\section{Refer with confidence}

Some patients may require multidisciplinary treatment that is beyond your professional remit, so in such cases, dental professionals could consider referring them to the award-winning team of clinicians at Ten Dental Facial.

Comprised of implantology experts, and specialists in restorative dentistry, periodontics, endodontics, and orthodontics, the team at Ten Dental Facial is highly experienced in treating complex cases.

They have all the necessary skills and knowledge to deliver consistently high quality care to every patient, including those with additional needs.

With multiple state-of-the-art clinics located in the heart of London, dental professionals can refer to Ten Dental Facial with confidence, knowing that patients will be returned to their professional care following completion of treatment.

More information is available by visiting www.tendental.com or calling 02033932623 .

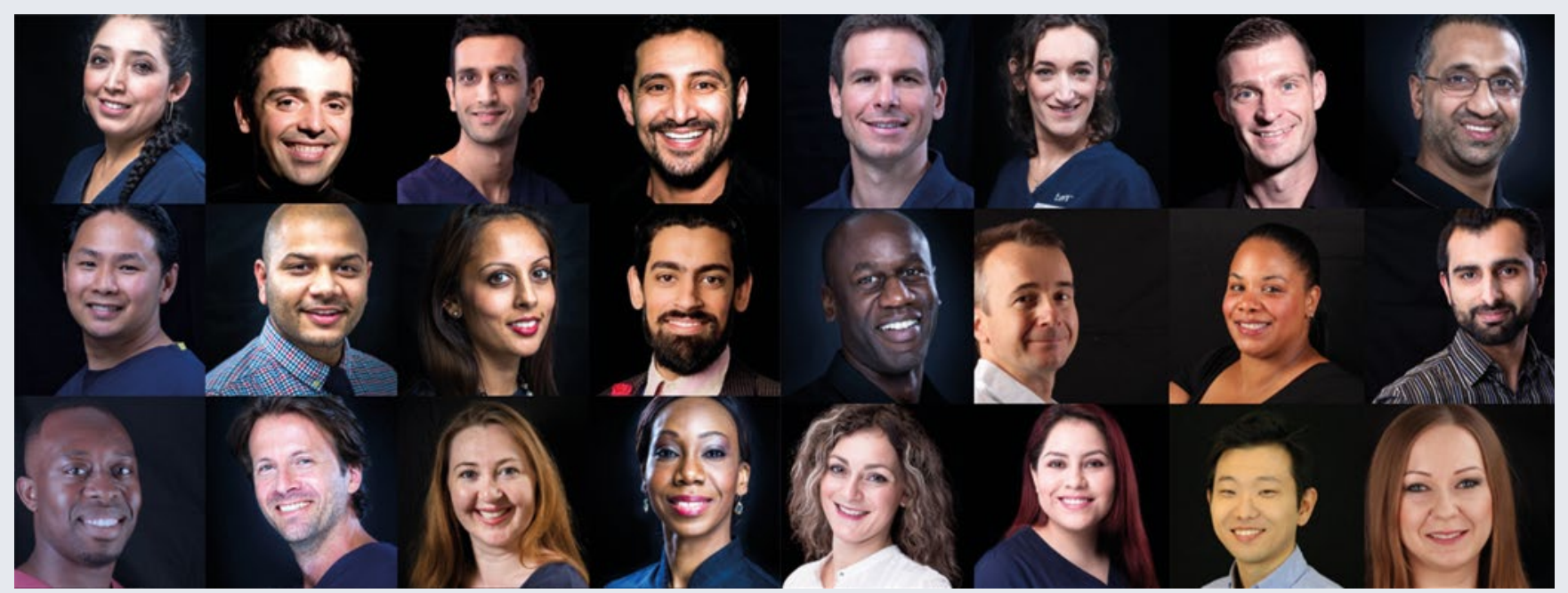

\section{The speedier solution}

Want to be able to take impressions fast and with no fuss? Try new Impregum Super Quick polyether impression material from 3M Oral Care.

Needing only 45 seconds of working time, the material sets in just two minutes, helping you to streamline your workflows and improve patient comfort thanks to its fresh minty taste.

Impregum Super Quick polyether impression material is also scannable with the current generations of scanners and compatible with a CAD/CAM workflow, helping to take your impressions to the next level.
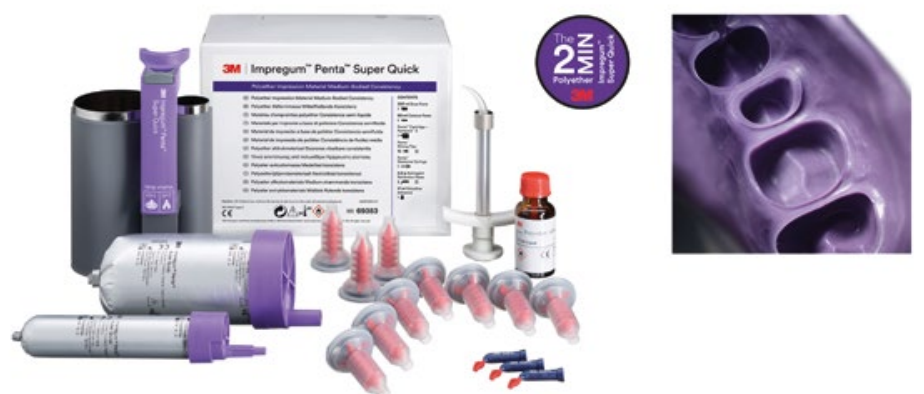

Find out more about Impregum Super Quick polyether impression material and request your demo by visiting www.3M.co.uk/ImpSQ.

$3 \mathrm{M}$ and Impregum are trademarks of the $3 \mathrm{M}$ Company.

\section{A clean, healthy mouth}

Dentistry accounts for nearly $10 \%$ of all antibiotic prescribing in the UKbut with good preventive measures, dental professionals can reduce levels of decay, one of the primary causes of abscesses that can lead to an infection.

The high-quality brushes from TANDEX can be recommended for daily use, along with the FLEXI interdental and the SOLO interspace brush to remove bacteria gently, yet efficiently. PREVENT GEL is also available to support good oral hygiene.

TANDEX prides itself on a commitment to functionality and quality and recommends dentists bring its products to their practices so patients can feel the difference.

More information on Tandex's range of products is available at www.tandex.dk or its facebook page www.facebook.com/pages/ Tandex-UK/234855250044190? fref=ts.

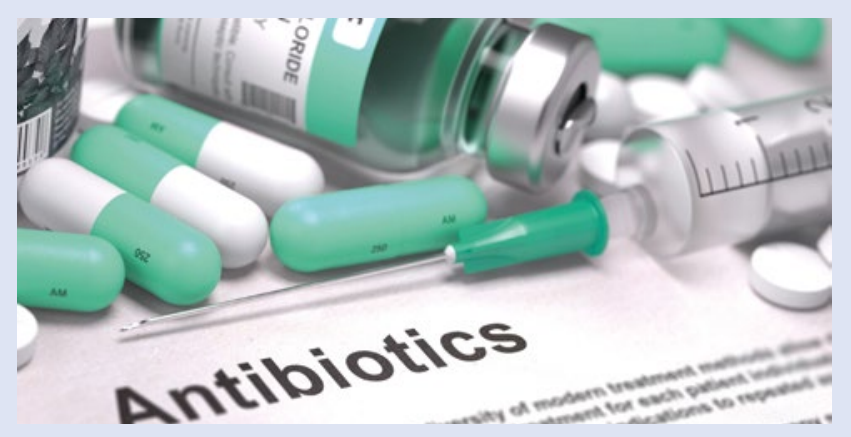

